\title{
Early knapping techniques do not necessitate cultural transmission
}

William D. Snyder, ${ }^{1 *}$ Jonathan S. Reeves, ${ }^{1,2}$ Claudio Tennie ${ }^{1,3 *}$

${ }^{1}$ Department of Early Prehistory and Quaternary Ecology, Eberhard Karls University of Tübingen, Tübingen, Germany

2 Technological Primates Research Group, Max Planck Institute for Evolutionary Anthropology, Leipzig, Germany

${ }^{3}$ Department of Human Evolution, Max Planck Institute for Evolutionary Anthropology, Leipzig, Germany

* Corresponding authors: William D. Snyder, wdspaleo@gmail.com; Claudio Tennie, claudio.tennie@uni-tuebingen.de

\begin{abstract}
Early stone tool production, or knapping, techniques are claimed to be the earliest evidence for cultural transmission in the human lineage. Previous experimental studies have trained human participants to knap in conditions involving opportunities for cultural transmission. Subsequent knapping was then interpreted as evidence for a necessity of the provided cultural transmission opportunities for these techniques. However, a valid necessity claim requires showing that individual learning alone cannot lead to early knapping techniques. Here, we tested human participants $(N=28)$ in cultural isolation for the individual learning of early knapping techniques by providing them with relevant raw materials and a puzzle task as motivation. 25 participants were technique-naïve according to post-test questionnaires, yet they individually learned early knapping techniques, therewith producing and using core- and flake tools. Early knapping techniques thus do not necessitate cultural transmission and could likewise have been individually derived among pre-modern hominins.
\end{abstract}

One-Sentence Summary: The early stone tool record is not unequivocal evidence for humanlike cultural transmission in pre-modern hominins.

Keywords: Social learning, cumulative culture, cultural transmission, stone tools, knapping experiments, baseline performance

Author contributions: Conceptualization: CT, WDS, JSR; Funding acquisition: CT; Methodology: CT, WDS, JSR; Project administration: WDS, CT; Resources: WDS, CT, JSR; Investigation: WDS; Visualization: WDS; Formal analysis: WDS, JSR; Supervision: CT; Writing - original draft: WDS; Writing - review \& editing: WDS, CT, JSR 


\section{Introduction}

Cumulative culture of know-how defines the human niche (Boyd, 2018; Boyd \& Richerson, 1996; Henrich, 2016; Tennie et al., 2020). The products and cognition resulting from this special type of culture enable the adaptive success of the human species (Boyd, 2018; Henrich, 2016). This type of culture requires particular social learning mechanisms, namely those that allow know-how information (Bandini et al., 2020; Tennie et al., 2020) to be passed on intact, and thereby to accumulate across generations until the accumulated know-how becomes impossible for single individuals to re-innovate (Boyd \& Richerson, 1996; Reindl et al., 2016). Despite its central significance, the origin of cumulative culture within the human lineage is not well-understood. Cumulative culture of know-how is argued to have emerged as early as the Oldowan industry (e.g., Caruana et al., 2013; Morgan et al., 2015; Schick \& Toth, 1994; Shipton \& Nielsen, 2015; Stout et al., 2019), a stone tool technology which first appeared around 2.6 million years ago (Ma) (Ambrose, 2001; Braun et al., 2019).

The central component of Oldowan technology was the use of percussion (e.g., with a hammerstone, anvil, or both) to create sharp edges on stone, typically via a mechanical process known as conchoidal fracture (Ambrose, 2001; Cotterell \& Kamminga, 1987; Toth, 1985). The repetition of this process within and across individuals resulted in assemblages of cores and detached pieces (thus, a "simple" core- and flake technology; Toth, 1985). Primarily the flakes, but potentially also some cores, would have been used as cutting tools for butchering and other foraging tasks (Ambrose, 2001; Toth, 1985; Wynn \& McGrew, 1989).

The technical know-how underlying Oldowan artefacts, despite their comparative technological simplicity (Harlacker, 2003; Stout et al., 2015, 2021), is claimed to have already been maintained by the same types of social learning used by living modern humans mechanisms able to transmit know-how that has culturally evolved beyond the individual reach (Caruana et al., 2013; Cataldo et al., 2018; Lombao et al., 2017; Morgan et al., 2015; Schick \& Toth, 1994; Shipton \& Nielsen, 2015; Stout et al., 2019). The main empirical basis for the claim that Oldowan artefacts provide evidence for such know-how copying skills are knapping experiments with human participants (e.g., Cataldo et al., 2018; Lombao et al., 2017; Morgan et al., 2015; Pargeter et al., 2021). These experiments showed a correlation between opportunities to learn how to knap via one or more of the social learning mechanisms (with models ranging from finished artefact forms to action demonstrations) known to be able to transmit know-how (Reindl et al., 2017) and improved knapping performances. These improved knapping performances under varied social learning conditions were then typically interpreted to infer that special learning mechanisms able to transmit know-how were necessary for learning how to knap and for creating artefacts resembling those from the Oldowan. These conclusions for modern humans were then extended to Oldowan tool production by extinct hominins, claiming that pre-modern hominins would have also required the same types of social learning mechanisms in order to reproduce knapping techniques and outcomes (Cataldo et al., 2018; Lombao et al., 2017; Morgan et al., 2015; Pargeter et al., 2021; Stout et al., 2019).

Additionally, previous studies have trained participants how to knap in abstraction from the inferred ultimate purpose of these artefacts - namely, tool use for extractive foraging and other tasks. In prehistory, expedient, least-effort toolmaking (Putt, 2015; Toth, 1985) is believed to have occurred when needed, e.g., to access nutrients. Instead, individuals in these knapping studies have been tested with the explicit, isolated goal of learning how to knap (with monetary compensation and personal interest in learning the skill being the main motivators, e.g., Stout, 2011; Stout et al., 2011). Only very rarely have such experiments connected knapping with goals of making usable tools, as in some studies where there was provision of materials for the minimal testing of the sharpness of flakes (Bisson, 2001; Stout \& Semaw, 2006; Stout et al., 2009). Consequently, earlier knapping studies potentially suffered from a lack of ecological relevance regarding tool use. Meanwhile, non-human knapping studies have solved this problem via the inclusion of baited puzzle boxes into testing (a simulation of extractive foraging; pioneered by Wright, 1972). Using such puzzle boxes creates a motivation for the 
production and subsequent use of cutting tools, without having to solely rely on social paradigms to motivate skill learning.

Most importantly, while earlier human knapping studies have clearly shown a correlation between improvement in some aspects of knapping and certain types of social learning (though patterns across studies are often contradictory; e.g., Cataldo et al., 2018; Lombao et al., 2017; Morgan et al., 2015; Putt et al., 2014), no study has tested whether cultural transmission of know-how is necessary. For example, it might be that various social learning mechanisms - as specifically expressed in modern humans - may merely speed up individual learning of knapping (some may even do so faster than others). This is an important caveat, as there are hypotheses, which posit that the knapping techniques from before and during the Oldowan (hereafter, early knapping techniques) should have been expressible by individuals in the full absence of social learning mechanisms able to transmit know-how - i.e., without cultural transmission (Tennie et al., 2016, 2017). Under this account, some types of social learning were likely still at play, but which only regulated the frequencies of such individual know-how re-innovations (Bandini \& Tennie, 2017) - creating "minimal cultures" (Buskell \& Tennie, 2022; Neadle et al., 2017). If true, the Oldowan would not mark the beginnings of modern human-like cumulative culture after all.

Earlier human knapping studies have not tested for the possibility of individual re-innovation of early knapping techniques (Tennie et al., 2016, 2017). To test whether early knapping techniques can be arrived at individually, the development of knapping skill must be examined in cultural isolation from the underlying know-how. Said cultural isolation can be achieved by the "baseline test method" in which knapping technique know-how is made culturally inaccessible to the test participants. By test design, any type of know-how transmission - be it via language, demonstrations, reverse engineering, or some other medium - must be strictly removed from the test situation (including before and during the experiment). This type of experimental paradigm is otherwise known as an Island Test (Tennie et al., 2016; Tomasello, 1999). Island Tests are the methodological benchmark for assessing the possibility of spontaneous and individual abilities to develop target know-how, such as early knapping techniques, without any opportunities for cultural transmission of know-how (Tennie et al., 2016; Tomasello, 1999).

Here, we present the results of an Island Test for early knapping techniques, run with human participants $\left(N=28 ; n_{\text {male }}=14, n_{\text {female }}=14\right)$. As in earlier non-human tests (Bandini et al., 2021; Toth et al., 1993; Wright, 1972), our human participants were given motivation to make and use cutting tools (a puzzle box openable by severing a rope) and access to the necessary raw materials for toolmaking (also known as know-what and know-where information; Bandini et al., 2020) (Fig. 1). Participants did not receive cultural information (demonstrated, spoken, written, or otherwise: Bandini et al., 2020; Tennie et al., 2020) related to stone tools, stone tool types or stone tool-related know-how, i.e., knapping techniques. We also controlled for participant naivety regarding stone tools and early knapping techniques via a questionnaire, but only after the test so that the questionnaire itself did not endanger the naivety status of the participants. Subjects that had previously been exposed to knapping techniques (naivety Levels 3 and 4) were regarded as non-naïve to the target know-how (Table 1). In these ways, we generated data from an Island Test condition for early knapping techniques. As it could not be established a priori whether and when participants would develop any knapping technique, we tested each participant in a sufficiently long (four-hour) session. Furthermore, all participants were tested alone in single sessions to exclude cultural transmission between individuals and to serve - in each case - as an independent replication of the results.

In this way, we were able to determine whether naïve humans could re-innovate early knapping technique(s) in the absence of cultural transmission of know-how. If naïve modern humans in our test can individually arrive upon any early knapping techniques - ideally, by inducing conchoidal fracture - and especially if they would develop all discussed techniques (passive hammer, bipolar, freehand, and projectile techniques) (de la Torre, 2019; Harmand 


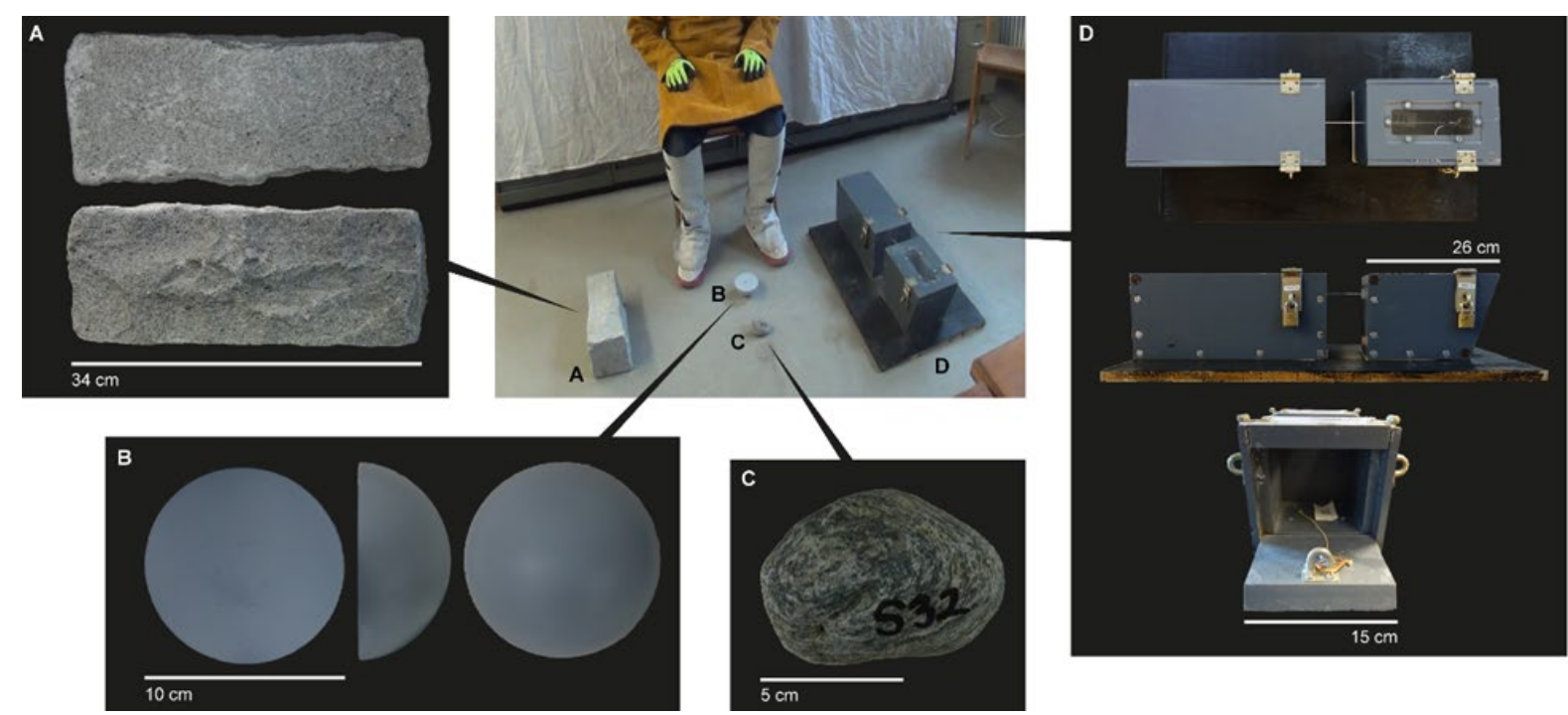

Figure 1. The experimental set-up. The experimental apparatus at testing start (here, for Participant 3; seated in the chair at the center, top), including (A) the granite block, (B), the painted glass hemisphere, (C), the river cobble, and (D) the puzzle box. This is the view as seen from Camera 2.

et al., 2015; Putt, 2015; Toth \& Schick, 2011, 2018; Wynn \& McGrew, 1989), then the hypothesis that early knapping techniques do not necessitate know-how copying would be supported. Simultaneously, the hypotheses that posit a necessity for cultural transmission of the know-how of early knapping techniques would become non-parsimonious.

\section{Materials and Methods}

Experimental design. Thirty participants were recruited via online and newspaper classified listings in a town in southwestern Germany. Two participants were tested but their data was excluded (due to safety concerns and experimenter error, respectively). A final sample of twenty-eight tested participants (fourteen female and fourteen male) are reported here. At the start of testing, each participant was provided with one spray-painted glass hemisphere (10 $\mathrm{cm}$ in diameter and $4 \mathrm{~cm}$ in height; note that hemispherical blank forms or "split cobbles" are known from the Oldowan: Toth, 1985), one locally sourced river cobble, and a large rectangular granite block (Fig. 1). Participants could receive replacements for the hemispheres and cobbles if the materials were deemed exhausted according to the participant's own evaluation. The puzzle box afforded the use of tools and consisted of a box with an enclosed reward accessible by severing a rope - a 'tendon' box (Bandini et al., 2021; Fig. 1). Due to the length of testing, both participant and experimenter(s) were given chairs. All tests were recorded using three separate digital camcorders, mounted on tripods at designated spots around the testing area. Participants were remunerated with $€ 12$ per hour plus any attained reward money.

Participants were not given any relevant information (verbal, written, or visual) about knapping or stone artefacts before or during the experiment. The participants were merely instructed to procure the reward from the puzzle box, using the available materials in the testing space. Participants were tested individually to further prevent any transmission of information and to ensure independent replication. The experimenter abided by a pre-determined script to avoid phrasing that might reveal the goals of the experiment (e.g., the experimenter referred to the cores, flakes, and other materials exclusively as "objects" and never specifically mentioned "cutting"). Overall, this setup allowed to test whether participants would produce and use glass or stone cutting tools by any variant of knapping technique, without compromising their naivety. This also ensured that the spontaneity of their technique innovations could be verified. 
Participation was contingent upon informed consent and fulfilment of eligibility criteria (ethical approval by the Ethics Committee for Psychological Research, University of Tübingen, confirmed on 12.06.2019). Pre-study information for the participants only indicated that this would be a study on "human problem-solving abilities", and did not mention stone tools, their form or manufacture techniques. This deception was necessary because transparency about the study's aims would have compromised the participants' naivety.

Due to health and safety regulations, participants and experimenter(s) wore safety gear and were not permitted to remove any of it while in the testing area. Each participant was tested for a maximum four-hour test (two tests ended early by participant request). Just prior to testing, participants were given explanations of the testing setup, including safety precautions and general study rules (e.g., no access to smart phones, no questions about specific solutions). The experimenter also clarified the mechanics of the puzzle box (i.e., that a visible rope prevented a door from being opened, thus further blocking access to the reward inside). After the initial study explanation, the experimenter signaled start, at which point the participant could begin pursuing solutions. If participants used a solution other than creating and using a cutting tool, the solution was noted and named live by the experimenter, and the participant was then instructed not to repeat this solution and to instead pursue other (unnamed) avenues of opening the puzzle box. The puzzle box was then reset and rebaited. The puzzle box was also reset and rebaited upon successful creation and usage of a cutting tool (either as a detached piece or cutting edge on a core).

Used detached pieces were retrieved by the experimenter and bagged and labelled, while successfully used edges on a core were visibly marked by the experimenter (with a pen) and verbally designated as unusable. This procedure simulated edge wear (as occurs in naturalistic conditions) and thus encouraged production of new edges. If the participant did not produce a cutting tool after two hours, they were provided an "impossible flake" (i.e., a tool with a functional shape - a wedge-like triangular prism made of glass and spray-pained - but impossible to recreate via knapping) for exactly one trial. Puzzle boxes were baited with paper slips designating monetary rewards. Participants received this monetary reward (as credit) for each time that they opened the puzzle box, regardless of the solution type.

After approximately five minutes of fruitless toolmaking attempts and/or total inaction (at any point during testing), participants were asked if they would like to receive a new blank. If participants requested a new blank (i.e., deemed exhaustion of the previous one), the old core and debitage were all collected and bagged with labels. Otherwise, collection, bagging and labelling of cores and debitage occurred at the end of the test.

In order to ensure that participants were naïve and remained naïve before and during testing, it was necessary to collect data on the participants' previous experience with stone tools after the test. Thus, once testing time was complete, participants filled out a questionnaire about their pre-study experiences - of any type - with stone tools.

Behaviors were coded live by the experimenter using a paper coding sheet. After the test ended, video recordings were processed (angle-selection and anonymization) and then coded. First, behavioral bouts (actions or action sequences with a definable start and end point) were identified as involving toolmaking, tool use, or simultaneous toolmaking and tool use. We coded events as potential toolmaking, both when actions did lead or could have led to fracture of an object and, with it, the creation of a potential cutting tool. Confirmed toolmaking events, on the other hand, were those where fracture of an object could be determined visually from the video recordings. The dichotomy of potential versus confirmed toolmaking was implemented as the intentions of the participants are not strictly known (unlike other studies, where the intentions are prescribed by the experimenters and therefore reasonably identifiable) (Cataldo et al., 2018; Lombao et al., 2017; Morgan et al., 2015; Pargeter et al., 2021; Stout et al., 2019). Toolmaking bouts were further assigned to one of four early knapping technique categories: freehand, passive hammer, bipolar, and projectile. Freehand, passive hammer, and bipolar techniques are all specifically associated with 
Oldowan technology (Toth \& Schick, 2011, 2018), while passive hammer (Harmand et al., 2015) and bipolar technique (Putt 2015; Wynn \& McGrew, 1989) are also associated with purported and hypothetical pre-Oldowan technologies. Projectile technique is not typically associated with the Oldowan proper but has been cited as a potential pre-Oldowan behavior due to its expression by the captive bonobo Kanzi and may have played a role in the innovation of knapping by naïve Oldowan hominins (Putt, 2015; Toth et al., 1993). We used a "constellation-based" approach for the assignment of toolmaking bouts to one of the technique categories; this method was based on earlier approaches (de la Torre \& Mora, 2005) but modified further so that technique classification was fully neutral in terms of intention, outcome, and mechanic (e.g., percussion, friction, pressure). In this approach, objects are identified using neutral terms like active, passive, auxiliary and target element, instead of already interpretative archaeological terms (e.g., 'hammer' or 'anvil'). If a participant used a technique in $\geq 50 \%$ of toolmaking events, this was defined as a preference.

For interobserver reliability calculation, a subset of data ( $n=7$ participants; $25.0 \%$ of participants) was coded by a hypothesis-unaware individual (J.K.) according to the above criteria. The coding from the experimenter and the reliability coder were then compared.

Participants' post-test questionnaire answers were used to rank the naivety of the participants, with the minimum rank of 0 for totally naïve individuals (with no theoretical or practical knowledge of stone tools) and a maximum rank of 4 for individuals with at least some previous hands-on experience with stone tools and knapping.

All detached pieces used by the participants as tools were immediately collected, labelled, and bagged for later analysis. Cores and all associated debitage (no matter how small) were collected and labelled either at the end of the test or if the core was exhausted. Used tools of any size and debitage elements equal to or above $2 \mathrm{~cm}$ in maximum dimension (Key \& Lycett, 2014) were subject to categorization as flakes or angular fragments (following Braun et al., 2019) and a full attributes analysis, two attributes of which are reported here. Platform depth (PD) was measured using digital calipers. For exterior platform angles (EPA), the analyst made three separate measurements of the angle with a goniometer and then averaged the three measurements (all reported EPAs are thus averaged values). Values for PD and EPA from the experimental flakes were compared with a reference dataset (Režek et al., 2018) from Oldowan sites (for our purposes, flakes that were labelled in this dataset as "Oldowan", "Developed Oldowan", and "Karari" were combined into one Oldowan technological category). For the cores, basic technological analysis was performed to elucidate the reduction strategies implemented by the participants (de la Torre, 2011) and the basic typology of the final cores (Leakey, 1972; Toth, 1985).

Statistical analysis. Statistical analyses and graph-building were performed using RStudio (RStudio Team, 2020). Due to non-normality of data distributions and incompatibility of sample sizes for the different naivety categories, non-parametric tests (Kruskal-Wallis test for oneway analysis of variance and Wilcoxon rank sum tests for pairwise comparisons) were used. Tests were two-sided (per the default setting in RStudio).

Cohen's kappa tests for interobserver reliability were used to measure agreement between the coding data of the experimenter (W.D.S.) with the coding data of the naïve secondary observer (J.K., see acknowledgements). Cohen's kappa tests were used on count data (frequency of a behavior or behavioral character) and Boolean data (presence/absence of a behavior or behavioral character). Similarly, a Cohen's kappa test was performed for interobserver reliability of flake identification, involving a comparison between the typing data from the experimenter (W.D.S.) and the hypothesis-naïve secondary observer (A.F., see acknowledgements). 
Table 1. Breakdown of participants by naivety level and behavioral outcomes. Reported values are the number of participants that fulfill both conditions (specified naivety level and specified behavioral outcome). The values reported in parentheses are those participants that also fulfill the criteria of the cell, but only did so after they had received the Impossible Flake. P6, P21, and P23 all performed knapping techniques in potential toolmaking events before receiving the Impossible Flake, but only P6 made and used cutting tools afterwards. P21 produced potential cutting tools without using them after receiving the Impossible Flake. P23 did so as well, but this was identifiable only from the artefactual outcomes and not via the coding of the video-recorded behavioral data.

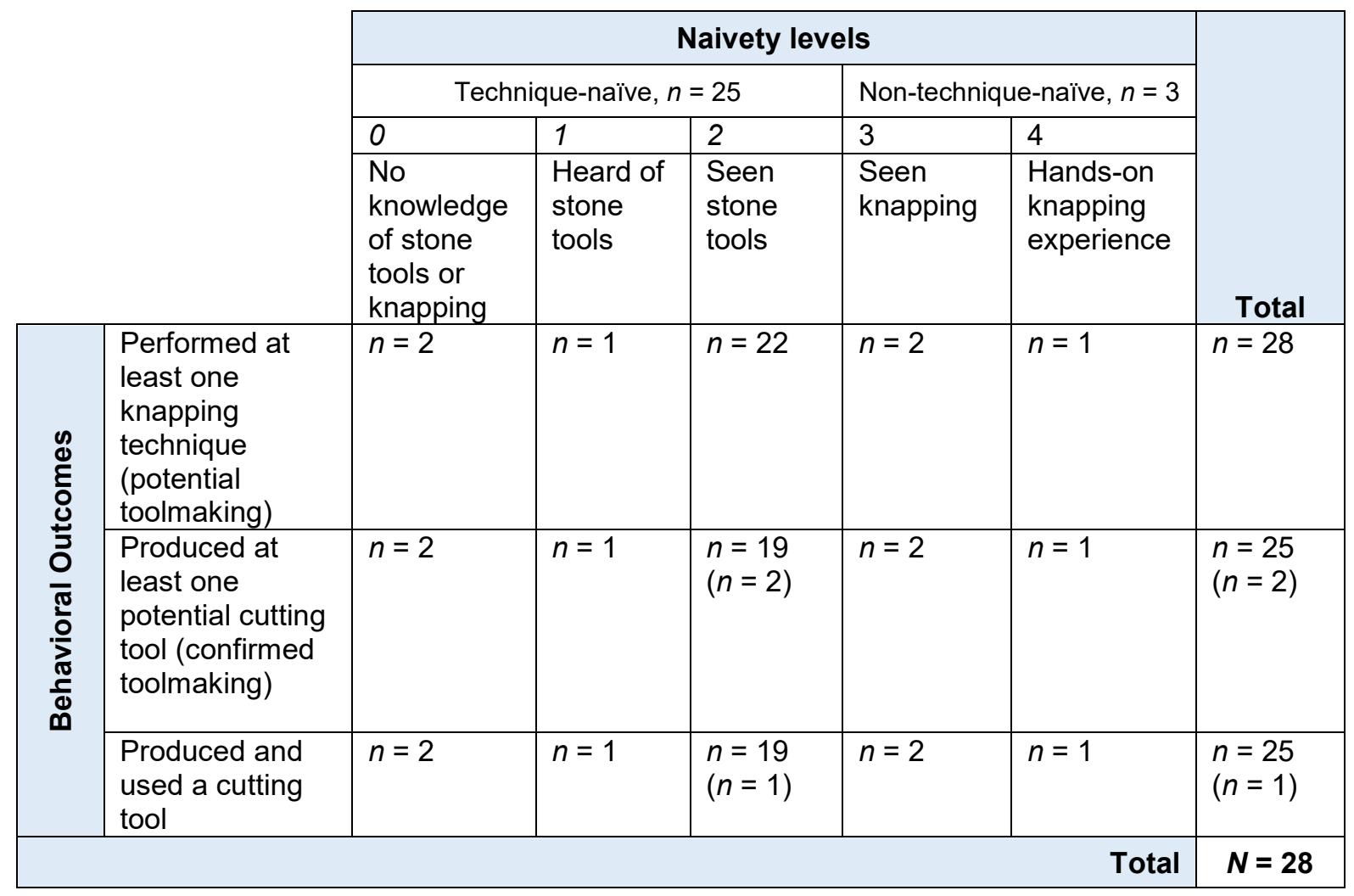

\section{Results}

As indicated by the post-test questionnaires, 25 of 28 participants were technique-naïve (i.e., had no knowledge of or previous experience with any knapping technique; naivety levels 0 to 2) (Table 1). Of these 25 technique-naïve participants, 22 participants had only seen stone tools before (naivety Level 2), one participant had heard of stone tools (Level 1), and two participants (Participants 11 and 14, henceforth P11 and P14) had been totally naïve concerning stone tools, stone tool types and knapping techniques (Level 0) prior to test. Despite the naïve nature of 25 participants regarding knapping techniques, all 25 of these spontaneously used knapping techniques, 22 of whom spontaneously produced and then used cutting tools (two others made potential cutting tools and one of those two used cutting tools after having been given the Impossible Flake, i.e., non-spontaneously). Importantly, both totally naïve (Level 0) participants individually developed early knapping techniques and used the resulting artefacts as cutting tools.

Overall, we recorded 1580 potential toolmaking events (i.e., where the actions therein could have or did leave to fracture of an object). Of these potential toolmaking events, $1095(69.3 \%)$ resulted in the fracture of an object and subsequent creation of potentially usable cutting edge (confirmed toolmaking events) (Fig. 2). It is important to note that, as typically occurs during knapping, a single bout sometimes produced more than one detached piece. The combined artefact assemblage at the end of this study consisted of 1599 objects, with flakes accounting for $73.3 \%$ of this total (1172 flakes produced from 33 glass cores) (Fig. 3). 


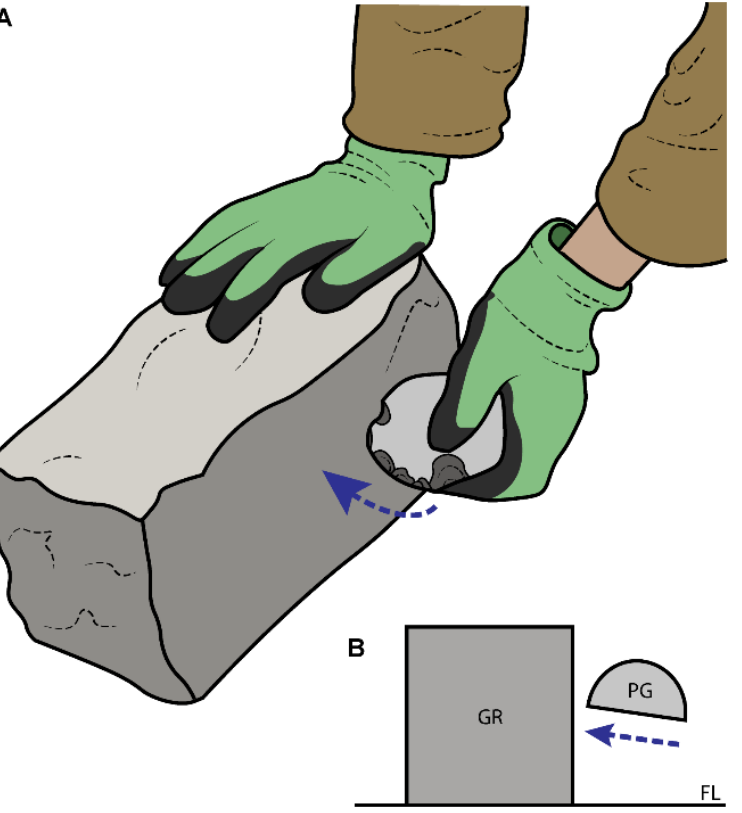

C

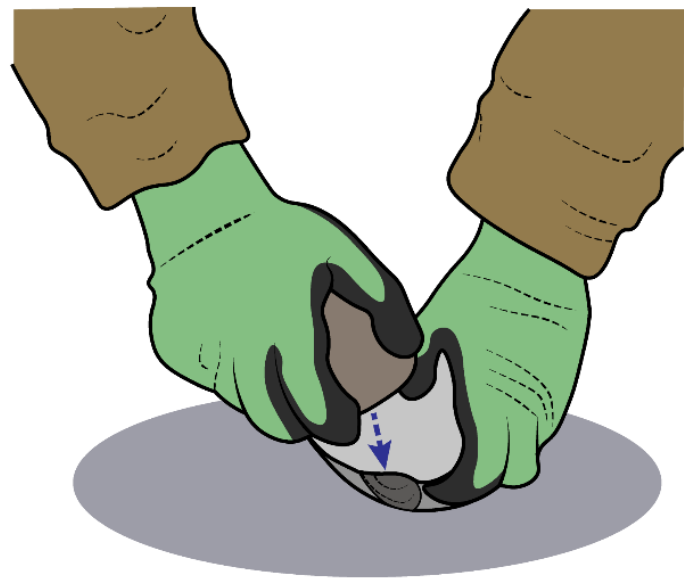

D

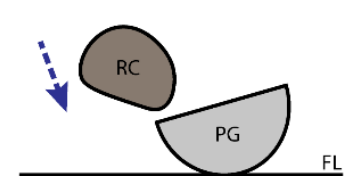

Figure 2. The preferred knapping techniques of the two totally naïve participants (Level 0). (A to B) Passive hammer technique used by P14 (Trial 45) shown from the perspective of Camera 2 (A) and in profile view (B). (C to D) Bipolar technique used by P11 (Trial 23) shown from the perspective of Camera 2 (C) and in profile view (D). Blue, dashed arrows represent the directionality of force of the active element. The abbreviations in the profile views ( $\boldsymbol{B}$ and $\boldsymbol{D})$ stand for the following: $G R$ is the granite block, $P G$ is the painted glass hemisphere, $R C$ is the river cobble, and $F L$ is the concrete floor.

All confirmed toolmaking events for the first naïve participant P11 ( $n=36,100.0 \%)$ involved the application of bipolar technique. The preferred approach (used in over half of the toolmaking bouts) (Fig. 2C to D) for P11 was to place the glass hemisphere directly on the concrete floor, stabilize the core (glass hemisphere) with one hand, and then strike it from above with the provided river cobble (as a hammer), using the other hand. When considering potential toolmaking events more broadly, P11 also showed evidence for both passive hammer $(n=1)$ and freehand $(n=1)$ techniques. As for products, P11's toolmaking primarily resulted in the production of flakes ( $n=35$ out of $N=41$ artefacts in P11's assemblage; Figs. 3 and 4).

The first technique used by the other totally naïve participant P14 was freehand $(n=1,1.9 \%)$, though nearly all confirmed toolmaking events for P14 $(n=53,98.1 \%)$ involved a variant of passive hammer technique (Fig. 2A to B), in which the outer edge of the glass hemisphere was struck against the side of the granite block. Regarding potential toolmaking events, P14 additionally engaged in bipolar technique $(n=1)$. Just as with P11, P14's toolmaking actions produced mostly flakes ( $n=61$ out of $N=77$ artefacts) (Figs. 3 and 4).

Among all 28 participants, twenty-two used more than one technique to successfully create sharp edges/cutting tools. All but one participant demonstrated a preference for one technique over other techniques. The total number of techniques expressed by individuals did not differ across naivety levels (Kruskal-Wallis test, $X^{2}=5.7528$, D.F. $=4, p=0.2184$ ).

The three early knapping techniques (passive hammer, bipolar, and freehand) reinnovated by the two totally naïve participants were also often used by other participants. The fourth coded category, projectile technique, though not shown by either totally naive participant, was developed by several other participants - all of whom were knapping technique-naive ( $n=1$ for Level 1 and $n=9$ for Level 2) (Fig. 2). 

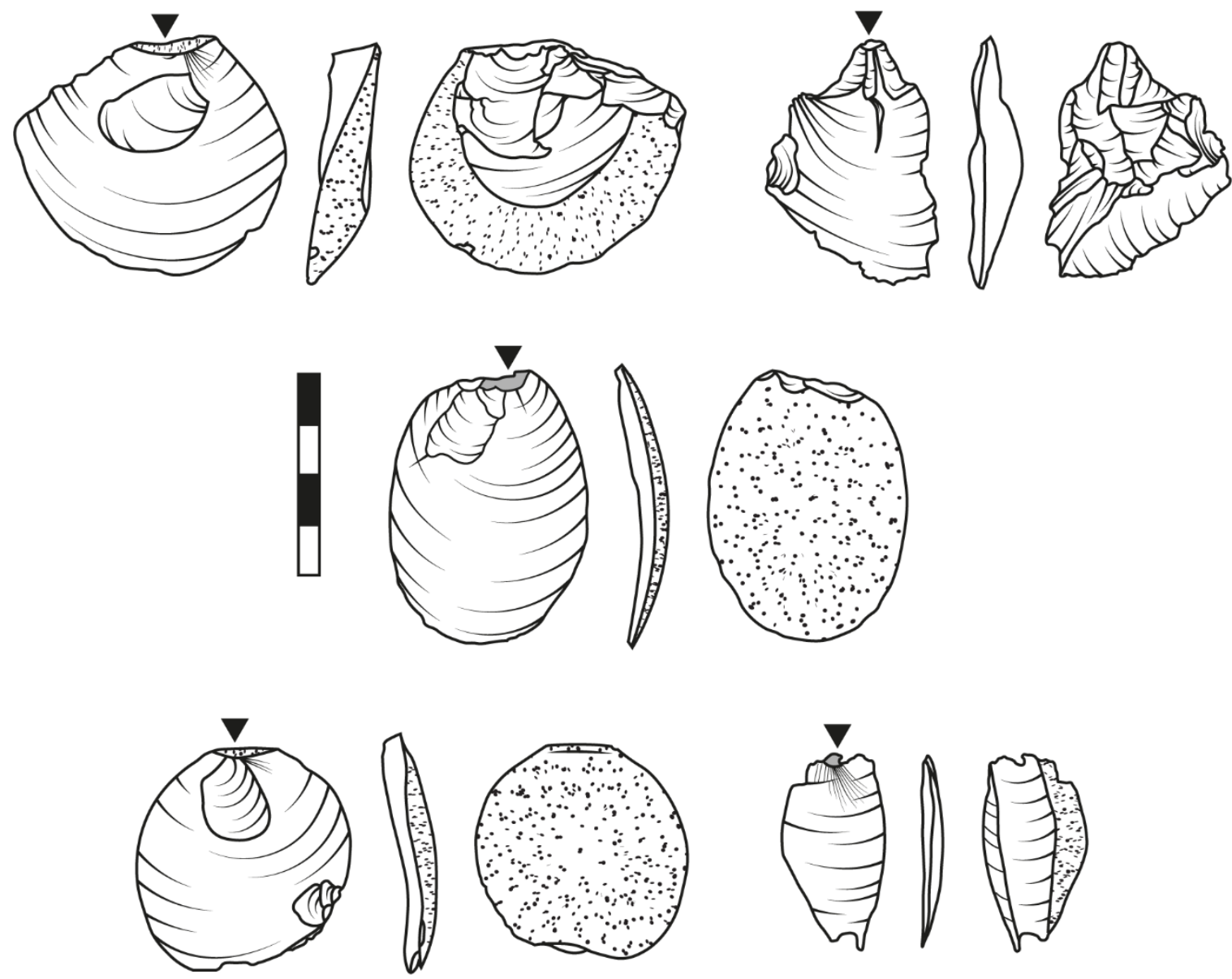

Figure 3. Technological illustrations of flakes. All flakes depicted here were made by individuals who had never before seen the process of stone toolmaking. All flakes were used successfully as cutting tools. Artefacts are shown in ventral, lateral, and dorsal perspective and are labelled by participant ID (e.g., P11), participant naivety level (e.g., LO), and the knapping technique that was used to produce the flake. Flakes are shown in platform-up orientation, with black triangles pointed at the approximate location of the point of percussion. Light grey areas indicate crushing events while dotted areas indicate cortex (the original painted surface of the glass hemispheres). Scale represents $4 \mathrm{~cm}$.

Across all attempts and across all participants, bipolar technique was the most frequent category of potential toolmaking ( 574 events, 36.3\%), followed by passive hammer (559 events, $35.4 \%$ ), freehand (410 events, $25.9 \%$ ), and projectile (37, $2.3 \%)$. In terms of individual preference for technique, the preferred technique in potential toolmaking events was most often bipolar technique $(n=10,35.7 \%)$, followed by passive hammer $(n=7,25.0 \%)$, anviloriented $(n=5,17.9 \%)$, freehand $(n=4,14.3 \%)$ and opportunistic (no technique preference, $n=2,7.1 \%$ ). As far as confirmed toolmaking events, bipolar technique was the most frequent (466 events, $42.6 \%$ ), followed by passive hammer $(398,36.3 \%)$, freehand $(226,20.6 \%)$, and projectile $(5,0.5 \%)$. Individualized preferences for techniques were distributed between bipolar $(n=13,48.1 \%)$, passive hammer $(n=10,37.0 \%)$, freehand $(n=3,11.1 \%)$ and opportunistic (no technique preference, $n=1,3.7 \%$ ).

The first instance of potential toolmaking generally occurred within the first hour of testing $\left(t^{-}\right.$ $=20$ mins 38 secs, s.d. $=25$ mins 13 secs) (Fig. 5), with the confirmed first toolmaking also being relatively expedient $\left(t^{-}=35\right.$ mins 58 secs, s.d. $=56$ mins 20 secs $)$. Three individuals (P1, P3, and P28) produced and used a cutting tool as their very first solution to the puzzle box during the test. One of the two totally naive participants, $\mathrm{P} 11$, produced their first cutting tool after 20 minutes and 52 seconds of testing time. The second of the two totally naive 

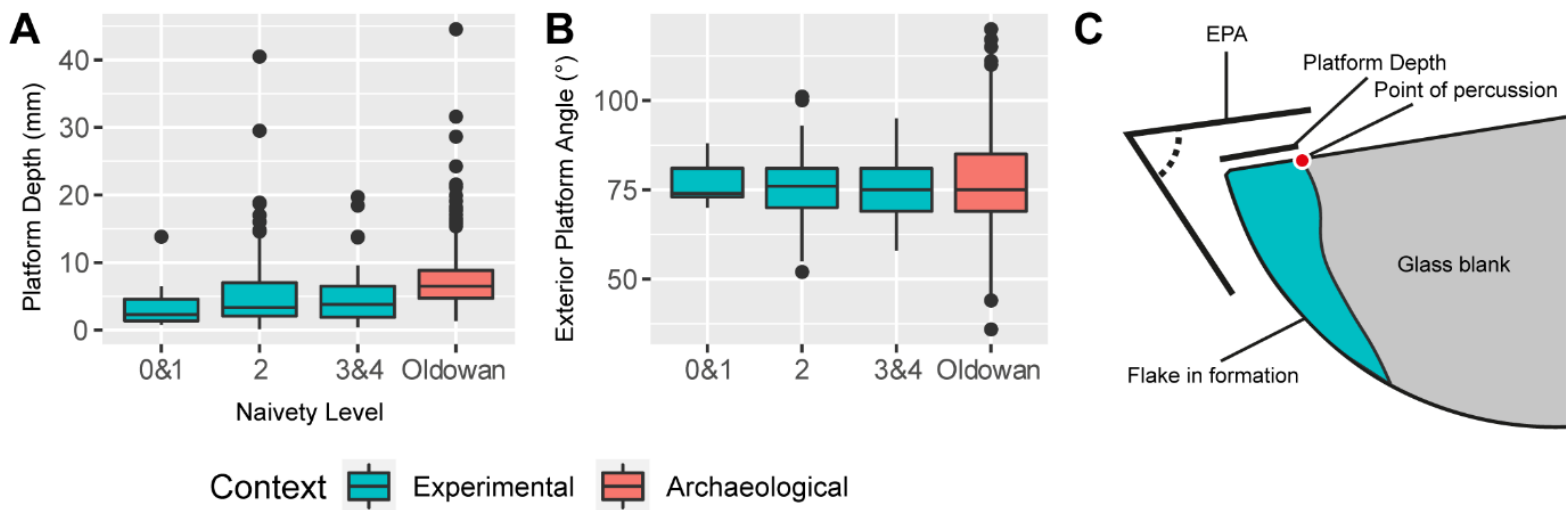

Figure 4. Metric comparison between experimental $(n=266)$ and archaeological flakes $(n=620)$. (A) Depth of platforms on experimental (left) and archaeological (Oldowan; right) flakes. (B) Exterior platform angle on experimental (left) and archaeological (Oldowan; left) flakes. (C) Schematic showing the formation of a flake by percussive knapping and the relationship to the aforementioned metrics.

participants, P14, produced their first cutting tool after 23 minutes and 23 seconds of testing time. There was no significant difference between naivety categories in terms of the timing of the first innovated potential toolmaking (Kruskal-Wallis test, $X^{2}=5.8528$, D.F. $=4, p=0.2104$ ) or of the first confirmed toolmaking (Kruskal-Wallis test, $X^{2}=5.3537$, D.F. $=4, p=0.2529$ ).

Participants across naivety categories did not differ in terms of the number of flakes produced (Kruskal-Wallis test, $X^{2}=3.0340, \quad D . F .=4, p=0.5522$ ). Experimental flakes possessed statistically shallower platforms (platform depth, PD) than Oldowan flakes (Kruskal-Wallis test, $X^{2}=111.96$, D.F. $\left.=3, p=2.2 \times 10-16\right)$ (Fig. 4A). All exterior platform angles (EPA) measured on experimental flakes (range of 52 to $101^{\circ}$ ) fell within the expected range for Oldowan flakes (Fig. 4B) (Režek et al., 2018). EPA was not significantly different between naivety groups and between naivety groups and Oldowan assemblages (Kruskal-Wallis test, $X^{2}=0.53688$, D.F. $=3$, $p=0.9107)$.

Patterns of removals (Fig. 4C) on the cores themselves varied across individuals. The most frequently appearing morphology were multifacial cores $(n=9)$, which were heavily reduced without clear evidence of systematic exploitation of specific knapping platforms. After this, the core type with the most frequent and identifiable pattern of removals were radial cores $(n=8)$ (Fig. 6). These cores involved the exploitation of the rounded surface of the hemisphere as a striking platform, causing mostly removals from the flat face that are directed at the midpoint of the circle. Knapping of a single exploitation surface or on two adjacent exploitation surfaces on one edge of the blank produced cores similar to unifacial $(n=5)$ and bifacial $(n=2)$ choppers, as well as heavy-duty $(n=2)$ scrapers. One Karari-style scraper $(n=1)$ (Harris and Isaac, 1976) was also produced by the complete exploitation and removal of the rounded surface of the blank.

\section{Discussion}

The origin of cultural transmission of know-how in the human lineage is a central focus in anthropological research. Previous claims based on correlational data from experiments in which humans learned to knap after cultural access to stone tools and/or knapping techniques align this origin with the earliest flake and core technologies (Cataldo et al., 2018; Lombao et al., 2017; Morgan et al., 2015,). Here, we tested for the spontaneous re-innovation of early knapping techniques in the absence of opportunities for cultural transmission of know-how. We validated our approach with post-test questionnaires, which established the techniquenaïve nature of the majority $(n=25)$ of our participants. We provided them only with motivation (a baited puzzle box) and the necessary raw materials for knapping (know-what and know- 


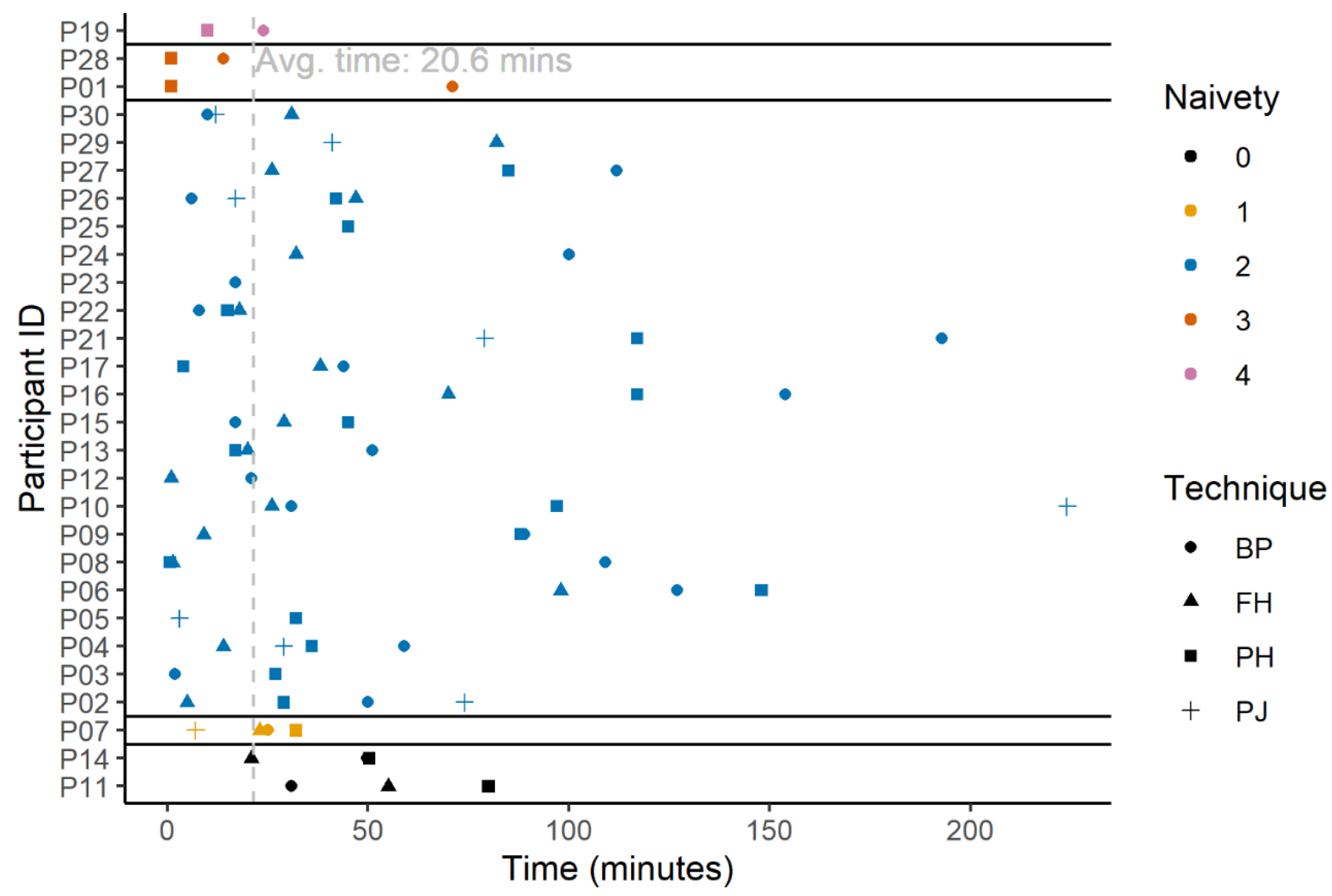

Figure 5. Timeline of first toolmaking innovations by technique. The timing of each innovation of a potential toolmaking technique for each participant (testing time elapsed at the start of the bout, in minutes). Shape of data points corresponds to the technique used in the behavioral bout and color of the data points corresponds to the naivety of the participant based on the post-test questionnaire (see legend). Grey, dashed, vertical line represents the average timing of the first innovation of any technique by each individual.

where: Bandini et al., 2020; Tennie et al., 2020). Despite most participants' naivety ${ }^{1}$ to knapping at the start of and the absence of know-how transmission during our experiment, all our technique-naive participants proved capable of individually re-innovating early knapping techniques. Two participants were found to be totally naïve to the very concept of stone tools - and yet even these participants re-innovated knapping and the usage of cutting tools. This finding calls for a re-interpretation of the conclusions from previous knapping studies regarding modern human knappers as well as with regard to pre-modern hominin knappers, seeing as these earlier studies did not truly test for technique-naive individual performances. Early knapping techniques, here, did not necessitate cultural transmission of know-how.

Contrary to claims that early knapping techniques must be culturally transmitted (Caruana et al., 2013; Morgan et al., 2015; Schick \& Toth, 1994; Shipton \& Nielsen, 2015; Stout et al., 2019), we found that all four of these techniques can be individually developed in the absence of such cultural transmission of know-how. The vast majority of our (mostly technique-naive) participants re-innovated early knapping techniques, and this included the totally naïve participants we tested, who had not only no previous cultural access to knapping techniques, but also no previous knowledge of stone tools and stone tool types. Overall, we detected re-

\footnotetext{
1 Participants in this study were "WEIRD" (referring to people from societies that are "Western, Educated, Industrialized, Rich, and Democratic": Henrich et al. 2010). This choice provides commensurability with previous knapping studies, which likewise used participants from WEIRD populations. WEIRD individuals are nonetheless appropriate for this type of study (i.e., an Island Test), since they are generally unlikely to be exposed to knapping experiences in their daily lives. Here, we controlled for remaining cultural transmission routes via the post-test questionnaire.
} 
innovation of all four of the discussed early knapping techniques by technique-naive participants (passive hammer, bipolar, freehand, and projectile; de la Torre, 2019; Harmand et al., 2015; Putt, 2015; Toth \& Schick, 2011, 2018; Wynn \& McGrew, 1989). Even the two technique-naïve participants who were also naive to stone tool concepts innovated three of the four knapping techniques (only projectile technique failed to appear in these two participants; see Fig. 2).

In previous knapping studies, there was a restricted expression of techniques, as participants' behaviors were often socially guided (and thus, limited), especially by prescribing them - via demonstrations or restrictions in the experimental setup (e.g., lack of potential anvils) specific toolmaking techniques that the participants were expected to express. Indeed, unless otherwise required by the testing procedure (i.e., for the study of passive hammer or bipolar techniques, e.g., Duke \& Pargeter, 2015; Putt, 2015), freehand technique was often the default, expected means of knapping in earlier experimental studies, despite existing evidence of other techniques throughout the record (de la Torre, 2019; Putt, 2015; Toth \& Schick, 2011, 2018). It is noteworthy that even where demonstrations were exclusively with freehand technique, there are still documented cases of apparent re-innovation of bipolar technique (Ferguson, 2003; Pargeter et al., 2021; Sternke and Sørensen, 2007). Overall, earlier studies seem to have triggered higher frequencies of expected techniques in participants via social means and/or the physical setup of the task at the expense of alternative techniques that were nevertheless in full range of participants' individual skill development. In our own study, with neither social nor physical guidance (or restrictions), participants were freed to express - and promptly expressed - a wider range of techniques. Also, there were many individuals in our study that switched between techniques, but these were likely a response to the dynamically changing form of the core (e.g., switching from freehand or passive hammer to bipolar when cores are more reduced and have fewer workable angles; Horta et al., 2022; Putt, 2015).

All technique-naive participants $(n=25)$ re-innovated knapping techniques, and among them, we found twenty cases of re-innovation of passive hammer technique, twenty-two cases for bipolar technique, twenty cases for freehand technique, and ten cases for projectile technique (re-innovation here meaning the first use of a particular early knapping technique by a single individual; see Fig. 5). Re-innovation by a single naïve subject - of any species- would logically suffice to show that any affected technique does not require cultural transmission. Therefore, with our many independent cases (i.e., in-study replications) of re-innovation, we provide ample empirical evidence that it is possible in principle that all four of the main proposed early knapping techniques (de la Torre, 2019; Harmand et al., 2015; Putt, 2015; Toth \& Schick, 2011, 2018; Wynn \& McGrew, 1989) can be individually re-innovated.

Flake production by conchoidal fracture is the hallmark physical component of Oldowan knapping (Ambrose, 2001; Cotterell \& Kamminga, 1987; Toth, 1985) and is also a feature that previous experimental studies have focused upon (Bandini et al., 2021; Cataldo et al., 2018; Morgan et al., 2015; Stout et al., 2015, 2021). The identification of a large portion of the artefacts produced in our study as conchoidally-produced flakes with EPAs in the same range as Oldowan material provides validity of our data to both the previous inexperienced knapper data under social learning conditions and the Oldowan record (Fig. 3B to C). That knapping by our naïve participants proved sufficient to generate Oldowan-like artefacts (see Figs. 3, 4, 6) should however be unsurprising, given the present interpretation that these core forms were the unintentional result of "least effort flaking" (Toth, 1985) - a view backed by recent empirical work on core form outcomes of purely stochastic knapping (Moore \& Perston, 2016). Our data provides further evidence that mental templates are not a necessary component of Oldowantype flaking (Moore \& Perston, 2016; Toth, 1985), with the affordances of the initial blank probably being most important in determining the patterns of reduction and the final core outcomes (e.g., the preponderance of radial cores from hemispherical blanks) (Toth, 1985).

External validity is a pertinent component of building an analogy between present experimental results and the prehistoric record we seek to understand (Eren et al., 2016; Lin et al., 2018). When designing knapping studies, there is an inherent tradeoff (Pargeter et al., 
2021) between experimental control on one hand, which typically improves precision, reliability and measurability, and naturalistic approaches on the other (i.e., using only those precise rock types and forms available to the hominins), which improve external validity at the expense of control. Here, we opted for high experimental control. We provided participants with hemispherical glass blanks, which were suitable for easy knapping (in terms of material and workable platforms), but we believe this was a justified choice in terms of external validity. Regarding the material, glass (albeit volcanic and not artificial glass) was exploited in prehistory, including in Oldowan contexts (Piperno et al., 2009), and in any case, glass conchoidally fractures not unlike other raw materials (Dogandžić et al., 2020). As for shape, hemispheres (or split cobbles) are also a known property of the Oldowan (e.g., Toth, 1985). In fact, our participants produced all the core outcomes that are expected from such hemispherical blanks, based on the Oldowan record (Toth, 1985).

Small differences between our experimental flakes and Oldowan flakes were detected. In this instance, the flakes from this study had shallower platforms than flakes from an Oldowan reference dataset (Režek et al., 2018). However, our study falls in line with the outcomes of previous human knapping studies, which have repeatedly shown that flakes made by inexperienced knappers are distinguishable from both flakes found in the archaeological record and those made by human expert knappers (Nonaka et al., 2010; Stout \& Semaw, 2006; Stout et al., 2009). We may therefore surmise that our participants entered a learning curve that is already known from previous human knapping studies, only via an alternative, individual, route. Indeed, even in group learning scenarios with ample social learning opportunities (and in two hours of testing), individuals in another knapping study did not achieve expert Oldowan knapping skill (Pargeter et al., 2021).

Given our findings, a more parsimonious explanation for the observed differentially increased performances in prior knapping studies involving know-how transmission opportunities (Cataldo et al., 2018; Lombao et al., 2017; Morgan et al., 2015; Pargeter et al., 2021; Putt et al., 2014) is that these opportunities might have merely sped up skill acquisition instead of 
being strictly necessary. In line with this is also the fact that acquisition of knapping techniques was very swift in these earlier studies (a few minutes sufficed, e.g., Morgan et al., 2015). Though we hypothesized that at least some naïve participants would re-innovate knapping techniques, the novelty of our approach meant that we were unable to predict how long human participants would require to individually re-innovate any knapping techniques, so we provided participants with an elongated learning time (four hours). These long learning times proved to be unnecessary, as technique re-innovation usually required only tens of minutes (Fig. 5). Future studies should however test whether technique-naïve humans, as tested in our study, would eventually reach expert-level performance on their own if simply afforded sufficient time and - with it - opportunities for individual learning. We tentatively hypothesize that such fully realized skill acquisition - expertise, perhaps to the level seen in the Oldowan - can be achieved solely via individual, hands-on knapping practice (cf. Harlacker, 2003; Stout and Chaminade, 2007; Stout et al., 2011). Ideally, future studies would investigate this hypothesis using a longer-term individual learning trajectory under Island Test conditions.

Contrary to the interpretation of data derived from earlier experimental studies that provided human learners with various types of cultural access to stone tools and/or their production techniques, cultural transmission proved unnecessary for the development early knapping techniques by human participants, and by extension, for the generation of flakes and artefactual assemblages with an Oldowan-like character. Our data thereby logically contradicts claims and assumptions that early knapping techniques necessitated cultural transmission of know-how in the past, as well (Caruana et al., 2013; Morgan et al., 2015; Shipton \& Nielsen, 2015; Schick \& Toth, 1994; Stout et al., 2019). Our findings logically show that - all - early knapping techniques can be individually developed by modern humans, which means that these techniques could have similarly been individually derived in the case of premodern hominins - as previously hypothesized (Tennie et al., 2016, 2017).

In light of these new findings, the interpretation of early knapping techniques and artefacts from the Oldowan warrants re-consideration. We advocate for a minimal culture model of Oldowan technology, wherein - as is proposed for non-human primate cultures (Bandini \& Tennie, 2017; Buskell \& Tennie, 2022; Neadle et al., 2017) - social learning does not transmit the know-how itself but instead affects the frequencies of serial re-innovations of a behavior within and across populations. The - likely multiple independent (Braun et al., 2019; de la Torre, 2019; Hovers, 2012) - origins of the Oldowan and the succession of later technological traditions would thus be better explained in terms of ecological and biological affordances. In such a scenario, instead of cultural transmission of know-how, other cognitive mechanisms (e.g., working memory, spatial and causal reasoning, motor coordination: cf. Haidle, 2010; Pradhan et al., 2012; Putt et al., 2017, 2019; Read et al., 2021; Stout and Chaminade, 2007; Stout et al., 2011; Stout et al., 2014, 2015; Tennie et al., 2017) would have played a proportionally larger role in early hominin technologies. Given the absence of spontaneous production or use of flakes via knapping in chimpanzees (Bandini et al., 2021), developments in some of these or related cognitive traits after the divergence of the human and Pan lineages would more cogently explain the emergence of the Oldowan and perhaps subsequent industries (Tennie et al., 2016, 2017).

On the grounds of the results presented herein, early core and flake technology can no longer be used as unequivocal evidence for an early onset of cultural transmission of know-how, suggesting a re-examination of the beginnings of cumulative cultural evolution of know-how. Consequently, the earliest unequivocal evidence for technique transmission, and with it, cumulative culture, should be pushed forward to a later time, the identification of which should be the focus of future experimental research. 
Acknowledgments: We particularly and greatly appreciate the valuable input we received throughout the research process from Shannon McPherron. Similarly, Elisa Bandini has played a key supporting role from the very beginning. We would also like to thank the following colleagues for their advice and support: Angel Blanco Lapaz, David Boysen, Katerina Harvati, Li Li, Nico Michiels, Alba Motes-Rodrigo, Damien Neadle, Jordy Orellana Figueroa, Eva Reindl, and Britt 5 Starkovich. Special thanks to James Keppeler for performing the interobserver reliability coding and to Armando Falcucci for performing the interobserver reliability flake identification.

Funding: European Research Council (ERC) Horizon 2020 research and innovation program (grant agreement $n^{\circ} 714658$; STONECULT project, CT). 
References

Ambrose, S. H. (2001). Paleolithic technology and human evolution. Science, 291(5509), 1748-1753.

Bandini, E., \& Tennie, C. (2017). Spontaneous reoccurrence of "scooping", a wild tooluse behaviour, in naïve chimpanzees. PeerJ, 5, e3814.

Bandini, E., Motes-Rodrigo, A., Steele, M. P., Rutz, C., \& Tennie, C. (2020). Examining the mechanisms underlying the acquisition of animal tool behaviour. Biology Letters, 16(6), 20200122.

Bandini, E., Motes-Rodrigo, A., Archer, W., Minchin, T., Axelsen, H., HernandezAguilar, R. A., McPherron, S. P., \& Tennie, C. (2021). Naïve, unenculturated chimpanzees fail to make and use flaked stone tools. Open Research Europe, $1,20$.

Bisson, M. S. (2001). Interview with a Neanderthal: An Experimental Approach for Reconstructing Scraper Production Rules, and their Implications for Imposed Form in Middle Palaeolithic Tools. Cambridge Archaeological Journal, 11(2), 165-184.

Boyd, R. (2018). A Different Kind of Animal: How Culture Transformed Our Species. Princeton University Press.

Boyd, R., \& Richerson, P. J. (1996, January). Why culture is common, but cultural evolution is rare. In Proceedings-British Academy (Vol. 88, pp. 77-94). Oxford University Press Inc.

Braun, D. R., Aldeias, V., Archer, W., Arrowsmith, J. R., Baraki, N., Campisano, C. J., Deino, A. L., DiMaggio, E. N., Dupont-Nivet, G., Engda, B., Feary, D. A., Garello, D. I., Kerfelew, Z., McPherron, S. P., Patterson, D. B., Reeves, J. S., Thompson, J. C., \& Reed, K. E. (2019). Earliest known Oldowan artifacts at $>2.58 \mathrm{Ma}$ from Ledi-Geraru, Ethiopia, highlight early technological diversity. Proceedings of the National Academy of Sciences, 201820177.

Buskell, A., \& Tennie, C. (2022). Mere recurrence and cumulative change at the margins. British Journal for the Philosophy of Science, In Press.

Caruana, M. V., d'Errico, F., \& Backwell, L. (2013). Early hominin social learning strategies underlying the use and production of bone and stone tools. In C. Sanz, J. Call, \& C. Boesch (Eds.), Tool Use in Animals (pp. 242-285). Cambridge University Press.

Cataldo, D. M., Migliano, A. B., \& Vinicius, L. (2018). Speech, stone tool-making and the evolution of language. PLOS ONE, 13(1), e0191071.

Cotterell, B., \& Kamminga, J. (1987). The Formation of Flakes. American Antiquity, 52(4), 675-708.

de la Torre, Ignacio. "The early stone age lithic assemblages of Gadeb (Ethiopia) and the developed Oldowan/early Acheulean in East Africa." Journal of Human Evolution 60.6 (2011): 768-812.

de la Torre, I. (2019). Searching for the emergence of stone tool making in eastern Africa. Proceedings of the National Academy of Sciences, 201906926. 
de la Torre, I., \& Mora, R. (2005). Technological Strategies in the Lower Pleistocene at Olduvai Beds I \& II. Etudes et Recherches Archeologiques de l'Universite de Liege.

Dogandžić, T., Abdolazadeh, A., Leader, G., Li, L., McPherron, S. P., Tennie, C., \& Dibble, H. L. (2020). The results of lithic experiments performed on glass cores are applicable to other raw materials. Archaeological and Anthropological Sciences, 12(2), 44.

Duke, H., \& Pargeter, J. (2015). Weaving simple solutions to complex problems: an experimental study of skill in bipolar cobble-splitting. Lithic Technology, 40(4), 349-365.

Eren, M. I., Lycett, S. J., Patten, R. J., Buchanan, B., Pargeter, J., \& O’Brien, M. J. (2016). Test, Model, and Method Validation: The Role of Experimental Stone Artifact Replication in Hypothesis-driven Archaeology. Ethnoarchaeology, 8(2), 103-136.

Ferguson, J. R. (2003). An experimental test of the conservation of raw material in flintknapping skill acquisition. Lithic Technology, 28(2), 113-131.

Haidle, M. N. (2010). Working-memory capacity and the evolution of modern cognitive potential: implications from animal and early human tool use. Current Anthropology, 51(S1), S149-S166.

Harlacker, L. (2003). Knowledge and know-how in the Oldowan: an experimental approach. In J. Apel \& K. Knutsson (Eds.), Skilled Production and Social Reproduction (pp. 219-243). Societas Archaeologica Upsaliensis.

Harmand, S., Lewis, J. E., Feibel, C. S., Lepre, C. J., Prat, S., Lenoble, A., Boës, X., Quinn, R. L., Brenet, M., Arroyo, A., Taylor, N., Clément, S., Daver, G., Brugal, J.-P., Leakey, L., Mortlock, R. A., Wright, J. D., Lokorodi, S., Kirwa, C., ... Roche, H. (2015). 3.3-million-year-old stone tools from Lomekwi 3, West Turkana, Kenya. Nature, 521(7552), 310-315.

Harris, J. W. K., \& Isaac, G. (1976). The Karari Industry: Early Pleistocene archaeological evidence from the terrain east of Lake Turkana, Kenya. Nature, 262(5564), 102-107.

Henrich, J. (2016). The Secret of Our Success: How Culture Is Driving Human Evolution, Domesticating Our Species, and Making Us Smarter. Princeton University Press.

Henrich, J., Heine, S. J., \& Norenzayan, A. (2010). The weirdest people in the world? Behavioral and Brain Sciences, 33(2-3), 61-83.

Horta, P., Bicho, N., \& Cascalheira, J. (2022). Lithic bipolar methods as an adaptive strategy through space and time. Journal of Archaeological Science: Reports, 41, 103263.

Hovers, E. (2012). Invention, Reinvention and Innovation: The Makings of Oldowan Lithic Technology. In Developments in Quaternary Sciences (Vol. 16, pp. 5168). Elsevier.

Key, A. J., \& Lycett, S. J. (2014). Are bigger flakes always better? An experimental assessment of flake size variation on cutting efficiency and loading. Journal of Archaeological Science, 41, 140-146. 
Leakey, M. D. (1971). Olduvai Gorge, Volume 3: Excavations in Beds I and II, 19601963. Cambridge University Press.

Lin, S. C., Rezek, Z., \& Dibble, H. L. (2018). Experimental Design and Experimental Inference in Stone Artifact Archaeology. Journal of Archaeological Method and Theory, 25(3), 663-688.

Lombao, D., Guardiola, M., \& Mosquera, M. (2017). Teaching to make stone tools: new experimental evidence supporting a technological hypothesis for the origins of language. Scientific Reports, 7(1), 1-14.

Moore, M. W., \& Perston, Y. (2016). Experimental Insights into the Cognitive Significance of Early Stone Tools. PLOS ONE, 11(7), e0158803.

Morgan, T. J. H., Uomini, N. T., Rendell, L. E., Chouinard-Thuly, L., Street, S. E., Lewis, H. M., Cross, C. P., Evans, C., Kearney, R., de la Torre, I., Whiten, A., \& Laland, K. N. (2015). Experimental evidence for the co-evolution of hominin tool-making teaching and language. Nature Communications, 6(1), 6029.

Neadle, D., Allritz, M., \& Tennie, C. (2017). Food cleaning in gorillas: Social learning is a possibility but not a necessity. PLOS ONE, 12(12), e0188866.

Nonaka, T., Bril, B., \& Rein, R. (2010). How do stone knappers predict and control the outcome of flaking? Implications for understanding early stone tool technology. Journal of Human Evolution, 59(2), 155-167.

Pargeter, J., Liu, C., Kilgore, M. B., Majoe, A., \& Stout, D. (2021). Testing the social, cognitive, and motor foundations of Paleolithic skill reproduction. OSF. https://osf.io/yebru/

Piperno, M., Collina, C., Gallotti, R., Raynal, J.-P., Kieffer, G., Bourdonnec, F.-X. le, Poupeau, G., \& Geraads, D. (2009). Obsidian exploitation and utilization during the Oldowan at Melka Kunture (Ethiopia). In E. Hovers \& D. R. Braun (Eds.), Interdisciplinary Approaches to the Oldowan (pp. 111-128). Springer Netherlands.

Pradhan, G. R., Tennie, C., \& van Schaik, C. P. (2012). Social organization and the evolution of cumulative technology in apes and hominins. Journal of Human Evolution, 63(1), 180-190.

Putt, S. S. (2015). The origins of stone tool reduction and the transition to knapping: an experimental approach. Journal of Archaeological Science: Reports, 2, 5160.

Putt, S. S., Woods, A. D., \& Franciscus, R. G. (2014). The role of verbal interaction during experimental bifacial stone tool manufacture. Lithic Technology, 39(2), 96-112.

Putt, S. S., Wijeakumar, S., Franciscus, R. G., \& Spencer, J. P. (2017). The functional brain networks that underlie Early Stone Age tool manufacture. Nature Human Behaviour, 1(6), 1-8.

Putt, S. S., Wijeakumar, S., \& Spencer, J. P. (2019). Prefrontal cortex activation supports the emergence of early stone age toolmaking skill. Neurolmage, 199, 57-69. 
Read, D. W., Manrique, H. M., \& Walker, M. J. (2022). On the working memory of humans and great apes: Strikingly similar or remarkably different? Neuroscience \& Biobehavioral Reviews, 134, 104496.

Reindl, E., Beck, S. R., Apperly, I. A., \& Tennie, C. (2016). Young children spontaneously invent wild great apes' tool-use behaviours. Proceedings of the Royal Society B: Biological Sciences, 283(1825), 20152402.

Reindl, E., Apperly, I. A., Beck, S. R., \& Tennie, C. (2017). Young children copy cumulative technological design in the absence of action information. Scientific Reports, 7(1), 1788.

Režek, Ž., Dibble, H. L., McPherron, S. P., Braun, D. R., \& Lin, S. C. (2018). Two million years of flaking stone and the evolutionary efficiency of stone tool technology. Nature Ecology \& Evolution, 2(4), 628-633.

RStudio Team. (2020). RStudio: Integrated Development for $R$ (Version 3.6.0) [Software]. http://www.rstudio.com/.

Schick, K. D., \& Toth, N. (1994). Making Silent Stones Speak: Human Evolution and the Dawn of Technology. Simon \& Schuster.

Shipton, C., \& Nielsen, M. (2015). Before Cumulative Culture: The Evolutionary Origins of Overimitation and Shared Intentionality. Human Nature, 26(3), 331-345.

Sternke, F., \& Sørensen, M. (2007). The identification of children's flintknapping products in Mesolithic Scandinavia. In McCartan, R. Schulting, G. Warren, \& P. C. Woodman (Eds.), Mesolithic Horizons. Papers Presented at the Seventh International Conference on the Mesolithic in Europe, Belfast 2005. Oxbow Books.

Stout, D. (2011). Stone toolmaking and the evolution of human culture and cognition. Philosophical Transactions of the Royal Society B: Biological Sciences, 366(1567), 1050-1059.

Stout, D., \& Chaminade, T. (2007). The evolutionary neuroscience of tool making. Neuropsychologia, 45(5), 1091-1100.

Stout, D., \& Semaw, S. (2006). Knapping skill of the earliest stone toolmakers: Insights from the study of modern human novices. In N. Toth \& K. Schick (Eds.), The Oldowan: Case Studies into the Earliest Stone Age (pp. 307-320). Stone Age Institute Press.

Stout, D., Schick, K., \& Toth, N. (2009). Understanding Oldowan knapping skill: An experimental study of skill acquisition in modern humans. In N. Toth \& K. Schick (Eds.), The Cutting Edge: New Approaches in the Archaeology of Human Origins (pp. 247-266). Stone Age Institute Press.

Stout, D., Passingham, R., Frith, C., Apel, J., \& Chaminade, T. (2011). Technology, expertise and social cognition in human evolution: Technology and cognition in human evolution. European Journal of Neuroscience, 33(7), 1328-1338.

Stout, D., Apel, J., Commander, J., \& Roberts, M. (2014). Late Acheulean technology and cognition at Boxgrove, UK. Journal of Archaeological Science, 41, 576590.

Stout, D., Hecht, E., Khreisheh, N., Bradley, B., \& Chaminade, T. (2015). Cognitive Demands of Lower Paleolithic Toolmaking. PLOS ONE, 10(4), e0121804. 
Stout, D., Rogers, M. J., Jaeggi, A. V., \& Semaw, S. (2019). Archaeology and the origins of human cumulative culture: a case study from the earliest Oldowan at Gona, Ethiopia. Current Anthropology, 60(3), 309-340.

Stout, D., Chaminade, T., Apel, J., Shafti, A., \& Faisal, A. A. (2021). The measurement, evolution, and neural representation of action grammars of human behavior. Scientific Reports, 11(1), 13720.

Tennie, C., Braun, D. R., Premo, L. S., \& McPherron, S. P. (2016). The Island Test for Cumulative Culture in the Paleolithic. In M. N. Haidle, N. J. Conard, \& M. Bolus (Eds.), The Nature of Culture (pp. 121-133). Springer Netherlands.

Tennie, C., Premo, L. S., Braun, D. R., \& McPherron, S. P. (2017). Early stone tools and cultural transmission: Resetting the null hypothesis. Current Anthropology, 58(5), 652-672.

Tennie, C., Bandini, E., Van Schaik, C. P., \& Hopper, L. M. (2020). The zone of latent solutions and its relevance to understanding ape cultures. Biology \& Philosophy, 35(5), 1-42.

Tomasello, M. (1999). The human adaptation for culture. Annual Review of Anthropology, 28(1), 509-529.

Toth, N. (1985). The Oldowan reassessed: a close look at early stone artifacts. Journal of Archaeological Science, 12(2), 101-120.

Toth, N., \& Schick, K. (2011). Factors affecting variability in Early Stone Age lithic assemblages: personal observations from actualistic studies. In J. M. Sept \& D. Pilbeam (Eds.), Casting the Net Wide: Papers in Honor of Glynn Isaac and His Approach to Human Origins Research (pp. 53-74). Oxbow Books.

Toth, N., \& Schick, K. (2018). An overview of the cognitive implications of the Oldowan Industrial Complex. Azania: Archaeological Research in Africa, 53(1), 3-39.

Toth, N., Schick, K. D., Savage-Rumbaugh, E. S., Sevcik, R. A., \& Rumbaugh, D. M. (1993). Pan the tool-maker: investigations into the stone tool-making and toolusing capabilities of a bonobo (Pan paniscus). Journal of Archaeological Science, 20(1), 81-91.

Wright, R. V. S. (1972). Imitative learning of a flaked stone technology - the case of an orangutan. Mankind, 8, 247-266.

Wynn, T., \& McGrew, W. C. (1989). An ape's view of the Oldowan. Man, 383-398. 